\title{
GESTIÓN PRESUPUESTARIA COMO FACTOR DETERMINANTE DE LA RENTABILIDAD EN EMPRESAS HOTELERAS DEL PERÚ (2012 - 2016)
}

\author{
BUDGETARY MANAGEMENT AS A DETERMINING FACTOR OF PROFITABILITY \\ IN HOTEL COMPANIES IN PERU (2012 - 2016)
}

\author{
Mara Pérez Carrasco \\ Universidad Nacional Mayor de San Marcos \\ Doctorado en Contabilidad con mención en Auditoria Superior-UNMSM \\ Correo electrónico: mpc@hotelessanagustin.com.pe
}

\section{RESUMEN}

Objetivo: El presupuesto, se constituye en una herramienta financiera de gestión importante para toda organización; en tal sentido el objetivo del presente trabajo de investigación es determinar si es la gestión presupuestaria factor determinante en la mejora de la rentabilidad de las empresas hoteleras en el Perú. Material: El material es de fuente primaria, obtenida en base a encuestas, entrevistas e información documental. El tipo de investigación, consideró la metodología descriptiva, explicativa, no experimental. Resultados: Los resultados determinan que los empresarios hoteleros nacionales, no observan herramientas básicas financieras, entre otros, la estructura presupuestal, que les permita una adecuada planificación financiera, hay un desconocimiento formativo intelectual empresarial, carecen de una disciplina permanente de fiscalización, limitando su accionar y competitividad y por ende su crecimiento patrimonial. Conclusiones: Se concluye, que la estrategia de administración financiera de la Cadena de Hoteles San Luis durante el periodo de análisis a través del uso de los presupuestos demuestra la consistencia de su aplicación al posibilitar que los objetivos de rentabilidad se vean alcanzados. Existe una relación direccional entre los procedimientos de auditoría de gestión y el tratamiento oportuno en la presentación de desviaciones presupuestales que podrían suscitarse durante la gestión.

Palabras clave: Estructura presupuestaria, auditoría de gestión, dirección hotelera, rentabilidad hotelera.

\begin{abstract}
Objective: The budget is an important financial management tool for any organization; in this sense, the objective of this research work is to determine if budget management is a determining factor in improving the profitability of hotel companies in Peru. Material: The material is from a primary source, obtained from the results of surveys, interviews and documentary information. The type of research considered the descriptive, explanatory, and non-experimental methodology. Results: The results determine that the national hotel businessmen in Peru fail to observe basic financial tools, among others, the budget structure, which could allow them an adequate financial planning. There is an absence of entrepreneurial training and lack of permanent discipline in auditing which limits their actions and competitiveness and therefore its patrimonial growth. Conclusions: It is concluded that the financial management strategy of the San Luis Hotel Chain during the period of analysis through the use of budget management demonstrates the consistency of its application to enable the profitability on the objectives to be achieved. There is a directional relationship between the management audit procedures and the timely treatment in the presentation of budget deviations that could arise during management.
\end{abstract}

Keywords: Budget structure, management audit, hotel management, hotel profitability. 


\section{INTRODUCCIÓN}

El turismo a nivel mundial, desde hace ya varias décadas ha venido mostrando un crecimiento sostenido; y en los últimos veinte años, esta actividad, ha llegado a ser muy representativa en algunos países respecto de su economía. No cabe pues ninguna duda, que el progreso socio económico de algunos países, se gestan en el desarrollo de esta actividad, que a la vez contribuye a reactivar un sin número de actividades relacionadas, que en suma generan un porcentaje de participación muy importante respecto del PBI de cada país. Según la revista especializada HVS/ HotelInvest-STR en "Previa del Panorama de la Hotelería en Sudamérica señala que pese a los problemas comunes que pueden afectar a todos los negocios hoteleros. Entre el 2011 y el 2015 se han integrado a la oferta 4,800 nuevos establecimientos de hospedaje".

En nuestro país, el turismo es la tercera actividad económica más importante, contribuyendo además al desarrollo social y económico y por ende a la reducción de pobreza, consecuentemente es catalogada por algunos expertos, cómo la actividad turística más rápida en estimular ingresos a la economía del país. La actividad de alojamiento y alimentación, es decir el negocio de los hoteles, en los últimos años ha obligado a los empresarios del rubro a elevar la calidad de sus servicios, observando estándares internacionales, que les permita ser más eficientes y competitivos.

El Informe denominado "Evolución de la Oferta Hotelera y aérea en el Perú” elaborado por la Dirección General de Investigación y estudios en turismo y artesanía DGIETA - Ministerio de Comercio Exterior y Turismo" Julio 2016, determina que, en el 2015, el Perú recibieron 3,5 millones de Turistas internacionales y que, en el 2016, la cifra se elevó a 307 millones. Una de las herramientas financieras, que permite optimizar la rentabilidad de estas empresas, sin lugar a dudas, son los presupuestos (Cárdenas, 2008).

Los estudios sobre el presupuesto de capital han hecho surgir la necesidad de investigar el costo de capital y el problema de coste de capital ha hecho surgir el problema óptimo de estructura financiera óptima (Suarez, 1970).

Del Rio Gonzales. (2007). Manual de Costos, Presupuestos y Adquisiciones y Abastecimiento. México: Cengage Learning Editores S.A. señala: "Presupuesto es la estimación programada, de manera sistemática, de las condiciones de operación y de los resultados a obtener por un organismo en un periodo determinado" (p. 34).

Según Robbins y Coulter. (2005). Administración. México: Pearson Educación, "El Presupuesto, está relacionado con una adecuada planificación. La Planificación tiene básicamente, dos propósitos fundamentales:

a. Protector: Minimiza riesgos e incertidumbres.

b. Afirmativo: Incrementa el nivel de efectividad organizativa mediante la coordinación" (p. 159).

El presente trabajo, busca analizar, investigar y determinar, como dentro de la nueva corriente y/o conceptos de los alojamientos a través de las cadenas de hoteles en el mundo, financieramente el uso de los presupuestos se convierte en un instrumento de 1era. Línea para el logro de esta nueva visión del negocio.

\section{MATERIAL Y MÉTODOS}

El trabajo es una investigación aplicada del tipo descriptiva, explicativa, no experimental que pretende demostrar que el uso de un adecuado presupuesto, en la gestión de las empresas hoteleras, coadyuvará al logro de los objetivos y metas, posibilitando el desarrollo y crecimiento de todas las actividades de las mismas, en el corto, mediano y largo plazo. En este contexto, se ha revisado las implicancias del presupuesto en las empresas hoteleras del país particularmente en la cadena de hoteles San Luis durante los años 2012 al 2016.

La utilización de métodos científicos en el desarrollo de un proceso de investigación, posibilita relacionar todos los preceptos teóricos, con la práctica. Significa ello, relacionar todos los conceptos teóricos ampliamente estudiados y validados, con la realidad de hechos concretos y reales. En este caso, la utilización de una herramienta de Gestión Financiera, como es el Presupuesto, observado y aplicado en la ejecución operativa que observa tanto los ingresos como los egresos de empresas dedicadas al rubro del hotelería.

El desarrollo de eventos en distintos ejercicios económicos conlleva el registro del comportamiento de las variables en estudio, en tal sentido el presente trabajo de investigación es descriptivo y es explicativo por que se pretende conocer los resultados de la aplicación del presupuesto en empresas hoteleras conociendo el compor- 
tamiento de otras variables relacionadas; Así, la variable "Aplicación del presupuesto en las empresas Hoteleras de Lima y Cuzco", implica la realización de las partidas presupuestales conforme a los datos estimados, a las políticas y directivas que sobre el particular emite el directorio a través de la gerencia general, consecuentemente se logra la maximización de la rentabilidad de las empresas, en este caso de las empresas hoteleras.

También se ha considerado que el presente estudio de investigación, es no experimental, pues será desarrollada con una estrategia que pretende analizar para responder a los problemas planteados, comprobar con tácita claridad los objetivos, experimentar la hipótesis general y específicas formuladas, sin la manipulación deliberada de variables, observando sólo el desarrollo de todos los procesos de manera minuciosa en su real ambiente.

Asimismo, por su dimensión temporal, es longitudinal, toda vez que el diseño que se utilizará, posibilitará observar en los períodos en analices que para tales efectos se estima de 05 años, los hechos tal y como se han ejecutado en su real contexto. Para tales efectos, en los citados períodos, la información fue analizada observando la metodología de recolección de datos consentidas la variables dependientes e independientes propuestas. Para tales efectos, los instrumentos utilizados fueron la Guía de entrevista estructurada; la posterior consolidación de datos; tabulación de hojas de cálculo y el análisis estadístico univariado y descriptivo de las variables.

Para la realización del presente trabajo de investigación, el objeto de estudio estuvo centrado en una importante cadena de hoteles, a la que hemos denominado Cadena Hotelera San Luis. La población de estudio, incluyó entre 280 a 300 aprox., de personal activo y socios estratégicos de la Cadena Hotelera San Luis. posee actualmente 09 establecimientos de hospedaje, ubicados en los siguientes lugares: 02 Hoteles en Lima, 01 hotel en Paracas, 01 Hotel en Arequipa, 03 Hoteles en Cusco y 02 Hoteles en el Valle de Urubamba. Adicionalmente, se adquirieron propiedades en los destinos turísticos de Puno, Chivay y Tarapoto.

Participaron activamente los funcionarios de línea y jefaturas de áreas; así como representantes de algunos bancos con los cuales la empresa tiene relación comercial; así como algunos clientes de agencias de viaje con pre- sencia internacional y proveedores que crecieron con la organización.

\section{RESULTADOS}

En los últimos años, se ha notado que muchos empresarios nacionales e internacionales, observan el negocio de hotelería como un atractivo de inversión; en lo que respecta a nacionales, es muy lento el crecimiento patrimonial, muchos de ellos crecen con recursos propios, aun cuando éstos les puedan significar muchos años. Las razones, pueden ser muchas, tales como:

> La falta de una adecuada planificación financiera, que les posibilite mirar a futuro de manera ordenada y disciplinada, utilizando todas las herramientas de gestión financiera, entre las cuales, sin lugar a dudas están "Los Presupuestos". Supervisada y Evaluada de manera permanente, para ver en primer lugar la curva de los ingresos y egresos, evaluar no sólo su cumplimiento, sino que éste a su vez, le otorgará un referente que llevará al análisis y preguntarse ¿por qué se logró? Y también ¿Por qué no se logró?, generar una disciplina permanente de fiscalización, donde también es importante la labor del Órgano de Control.

> Esta falta de conocimiento, también evidencia una persistencia en el empresario nacional a ser reaccionario a la utilización del endeudamiento a través de las entidades financieras, prefieren crecer con recursos propios, limitando la posibilidad de crecer con riesgo.

> Pueden ser muchos los factores, pero básicamente el desconocimiento formativo intelectual empresarial, marca la diferencia para enfrentar la competencia internacional.

Conforme a la metodología de investigación utilizada, aplicada a la población y otros relacionados a la Cadena de Hoteles San Luis se arribaron a los siguientes resultados:

$1^{\circ}$ "La eficiencia en la gestión de los ingresos por ventas presupuestadas, están en relación directa a niveles adecuados de retorno financiero en las empresas hoteleras". 
Tabla 1

Resultados Estadísticos Consolidados Para La Prueba De La Primera Hipótesis.

\begin{tabular}{|lccc|}
\hline PREGUNTA & SI & NO & TOTAL \\
\hline PREGUNTA 1 & 70 & 28 & 98 \\
\hline PREGUNTA 2 & 71 & 27 & 98 \\
\hline PREGUNTA 3 & 70 & 28 & 98 \\
\hline PREGUNTA 4 & 75 & 23 & 98 \\
\hline PREGUNTA 5 & 64 & 34 & 98 \\
\hline PREGUNTA 6 & 66 & 32 & 98 \\
\hline PREGUNTA 7 & 70 & 28 & 98 \\
\hline PREGUNTA 8 & 25 & 73 & 98 \\
\hline PREGUNTA 9 & 68 & 30 & 98 \\
\hline TOTAL & $\mathbf{5 7 9}$ & $\mathbf{3 0 3}$ & $\mathbf{8 8 2}$ \\
\hline
\end{tabular}

Fuente: Elaboración propia.

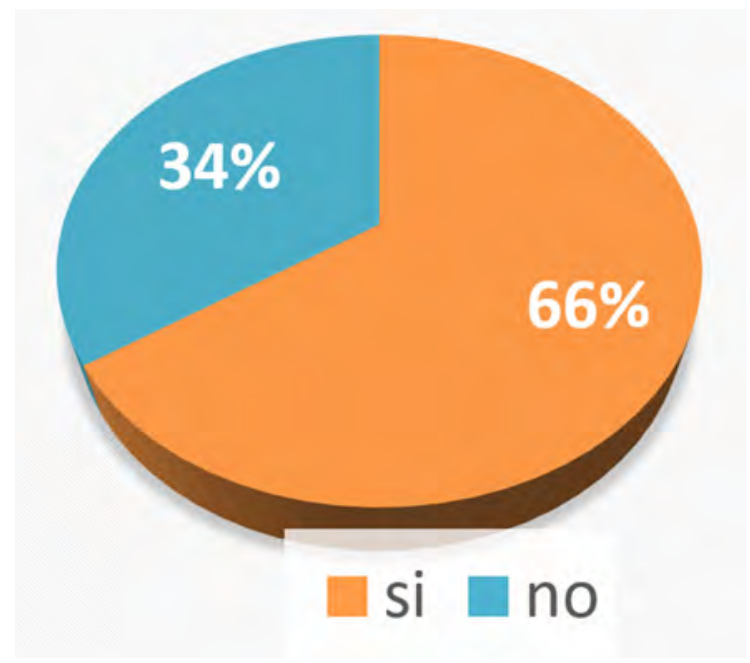

Figura 1. Nivel De Aceptación Por Resultados De Encuestas. Fuente: Elaboración propia.

De la tabulación efectuada a los resultados de las encuestas se aprecia que en su totalidad las respuestas validan la hipótesis planteada. Efectivamente, el uso de los presupuestos en la cadena de hoteles San Luis, vienen contribuyendo al crecimiento sostenido desde su consti- tución, posibilitando que la alta dirección pueda tener la seguridad que los proyectos acotados en su Planeamiento de Gestión Estratégica, se ejecute sin mayores contratiempos, en mérito a la rentabilidad presupuestada, que al final se cumple, pues las variaciones para alcanzar al 100\% 
el cumplimiento, están dentro de los rangos que podríamos denominar "contingencias aceptables", no excediéndose en todos los años que se evaluaron el porcentaje del $10 \%$, respecto del presupuesto esperado.

La evaluación presupuestal, incidió positivamente en todos los canales de ingresos de la organización, tales como: Hospedaje, alimentos y bebidas, servicio de teléfono, lavado de ropa, alquiler de equipos, alquiler de salones, catering etc.

La incidencia del presupuesto, estuvo básicamente centrada en fijar los parámetros de ejecución que posibilitaron el uso adecuado y racional de los insumos necesarios para brindar un servicio de calidad. El uso disciplinado en la ejecución de gastos, permitió mejorar al posibilitar los recursos esperados, la innovación permanente en la presentación del producto.

Asimismo, para efectos de los ingresos, si bien hay un presupuesto general, también hay un presupuesto a detalle por canal de ingresos, ya sea estos receptivos, corporativos, on line, GDS, OTAS etc. El presupuesto, en el tiempo fue aplicándose a medida, tanto para generar los ingresos, como para fijar los márgenes de gastos dependiendo de los ingresos que se esperaban alcanzar.

Esta disciplina en el uso del presupuesto, permite que la gestión se lleve de manera ordenada, posibilitando mejorar el producto en su generalidad, situación que ha permitido que una empresa nacional, tenga un buen posicio- namiento en el mercado hotelero, por muchos factores, entre ellos la calidad del servicio que ofrece, validado en los mayores comentarios positivos que se generan en las redes y en la permanente utilización de los servicios que oferta, de tal forma que sus ingresos siempre están en permanente crecimiento.

Adicionalmente, se observa la fidelización de sus clientes $y / u$ operadores turísticos, evidenciado en que se ha podido apreciar que en el transcurrir de los años continúan apoyándose mutuamente, existiendo un clima de cordialidad y confianza para derivar a sus clientes a la organización materia de análisis, siendo que además es el estado a través de sus instituciones que hacen uso del servicio, por la garantía que brinda la organización, que entre otros, cuenta con la certificación de restaurante saludable, en todos los establecimientos que posee la organización.

Esta forma de gestión, que observa al presupuesto como una herramienta financiera importante para el logro de los objetivos planteados, se refrenda con los resultados de las respuestas expuestas por los actores que tienen relación directa e indirectamente con la gestión de la organización.

$2^{\circ}$ "La eficaz programación de la inversión a través del mejoramiento de los servicios y de la infraestructura, está en relación directa a los niveles de gasto, mejorando la rentabilidad de las empresas hoteleras".

Tabla 2.

Resultados Estadísticos Consolidados Para La Prueba De La Segunda Hipótesis.

\begin{tabular}{|cccc|}
\hline PREGUNTA & SI & NO & TOTAL \\
\hline PREGUNTA 10 & 64 & 34 & 98 \\
\hline PREGUNTA 11 & 69 & 29 & 98 \\
\hline PREGUNTA 12 & 84 & 14 & 98 \\
\hline PREGUNTA 13 & 98 & 00 & 98 \\
\hline PREGUNTA 14 & 78 & 20 & 98 \\
\hline PREGUNTA 15 & 75 & 23 & 98 \\
\hline PREGUNTA 16 & 74 & 24 & 98 \\
\hline PREGUNTA 17 & 98 & 00 & 98 \\
\hline PREGUNTA 18 & 98 & 00 & 98 \\
\hline TOTAL & $\mathbf{7 3 8}$ & $\mathbf{1 4 4}$ & $\mathbf{8 8 2}$ \\
\hline
\end{tabular}

Fuente: Elaboración propia. 
En el siguiente cuadro se aprecia que la gran mayoría de encuestados expusieron de diferentes maneras su conformidad respecto a las preguntas formuladas, en este caso
738 respuestas que representa el $83,67 \%$ del total; De otro lado, 144 respuestas precisaron no estar de acuerdo, significando $16,33 \%$ del total.

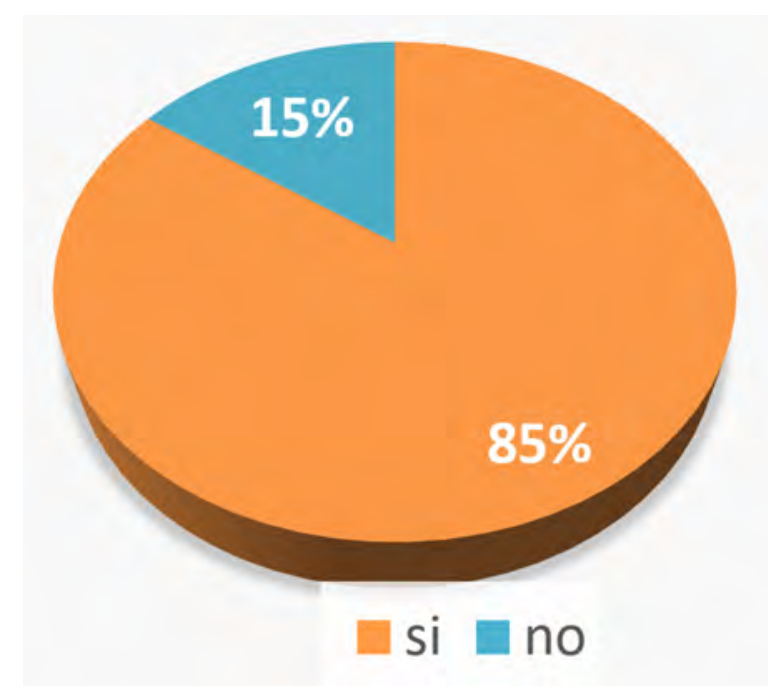

Figura 2. Nivel De Aceptación Por Resultados De Encuestas (2). Fuente: Elaboración propia.

$\mathrm{Al}$ respecto, cuando se relaciona recursos humanos - Incremento de rentabilidad - reducción de gastos corrientes, reducción de inversiones operativas, mejora costo/beneficio, podemos inferir de lo que hemos observado en el desarrollo de nuestro trabajo, en primer lugar que es política empresarial el buen trato, permanente capacitación y el adecuado ambiente laboral al interior de la organización, por cuanto según refieren, la empresa otorga un servicio directo al cliente, que no concluye con la entrega de un producto final, sino con la entrega de un producto permanente, que puede ser de $24,48,36$ horas y en algunos casos de semanas. El presupuesto, al establecer con antelación los ingresos, ayuda a la distribución adecuada del personal, evitando los sobrecostos, se debe tener en cuenta que el gasto laboral es uno de los más importantes en la organización.

Esta cordial relación que se da en su gran mayoría, coadyuva con el cuidado de las herramientas que se utiliza para el otorgamiento de los servicios, extendiendo razo- nablemente el tiempo de vida útil de los mismos, por ende, se reducen los gastos por inversiones operativas. Aporte mucho, el cumplimiento de sus compromisos y concesiones que otorga la organización a sus colaboradores, este accionar se garantiza por cuanto es una organización muy disciplinada. La diligencia laboral que en su gran mayoría el personal observa en su accionar diario, posibilita ejecutar inversiones en equipos muy onerosos, que coadyuvan al servicio de calidad que oferta la organización.

Con relación a la programación de compras, el presupuesto más allá del aporte que posibilita con las compras permanentes, propias del servicio diario, las que al final se ven reflejadas en el costo de ventas, al presupuestar los ingresos, pues con anticipación la parte operativa, prevé las adquisiciones de insumos, evitando los excesos, también considera el Cuadro de Adquisiciones anual, elaborado por todas las jefaturas operativas lideradas por sus administraciones, consigna mensualmente todas las adquisiciones que se harán en el año en beneficio de garantizar el 
servicio, permitiendo que el área de compras prevea con anticipación las adquisiciones, posibilitando que estas se ejecuten en las mejores condiciones, de calidad, cantidad, oportunidad y costo. $3^{\circ}$ "La auditoría de gestión, está en relación inversa a las desviaciones presupuestales que afecten la rentabilidad de las empresas hoteleras".

Tabla 3.

Resultados Estadísticos Consolidados Para La Prueba De La Tercera Hipótesis.

\begin{tabular}{|cccc|}
\hline PREGUNTA & SI & NO & TOTAL \\
\hline PREGUNTA 19 & 69 & 29 & 98 \\
\hline PREGUNTA 20 & 66 & 32 & 98 \\
\hline PREGUNTA 21 & 60 & 38 & 98 \\
\hline PREGUNTA 22 & 64 & 34 & 98 \\
\hline TOTAL & $\mathbf{2 5 9}$ & $\mathbf{1 3 3}$ & $\mathbf{3 9 2}$ \\
\hline
\end{tabular}

Fuente: Elaboración propia.

La representación gráfica señala que, de las 392 respuestas a las preguntas formuladas, 259 que representan el $66,07 \%$ del total, opinaron que si estaban de acuerdo con los planteamientos formulados respecto de la importan- cia de la auditoría de gestión y su importancia relacionada con el cumplimiento presupuestal; y 133 respuestas que representan el 33,93\% manifestaron no estar de acuerdo.

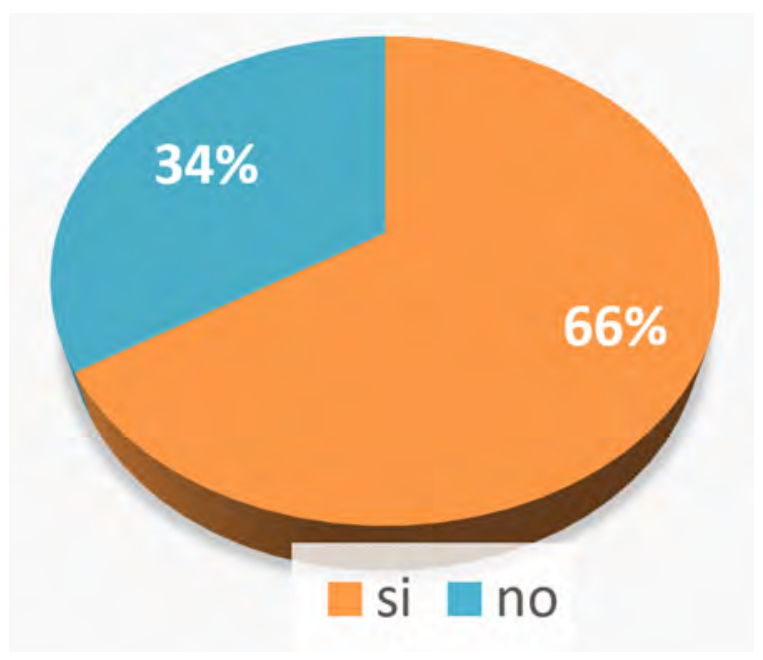

Figura 3. Nivel De Aceptación Por Resultados De Encuestas. Fuente: Elaboración propia. 
Al respecto, con la misma disciplina que los funcionarios comprometidos con la gestión de la organización observan el presupuesto, también se pudo observar la importancia de la aplicación de la auditoría de gestión como medio para mejorar la organización y por ende obtener mejores niveles de rentabilidad. Si bien el presupuesto coadyuva mucho a la gestión, direccionándola con antelación a la ejecución, la auditoría de gestión y financiera, colabora con la supervisión en el cumplimiento y desempeño de todas las actividades, sean estas operativas y/o administrativas. La auditoría de gestión y la auditoría financiera, inciden positivamente en la reducción de riesgos operativos y financieros al interior de la organización, coadyuvando en el cumplimiento del presupuesto, posibilitando lograr la rentabilidad esperada que permita tomar decisiones al Directorio.

\section{Prueba de hipótesis}

Resultado esperado de la aplicación Chi cuadrado sobre las encuestas realizadas:

Xexp. $\Sigma=6,56+18,38+13,41+36,50+2,55+7,22=84,62$

Cálculo del valor de la tabla Chi cuadrado:

$$
\begin{aligned}
& \mathrm{K}=(\text { Número de filas }-1) \times(\text { Número de columnas }-1) \\
& \mathrm{K}=(3-1) \times(2-1)=2
\end{aligned}
$$

\section{Nivel de Significancia}

Para el presente caso se toma el nivel de Significancia de $0,05 \%$, que indica que hay una probabilidad de $0,95 \%$ de que la hipótesis nula sea verdadera.

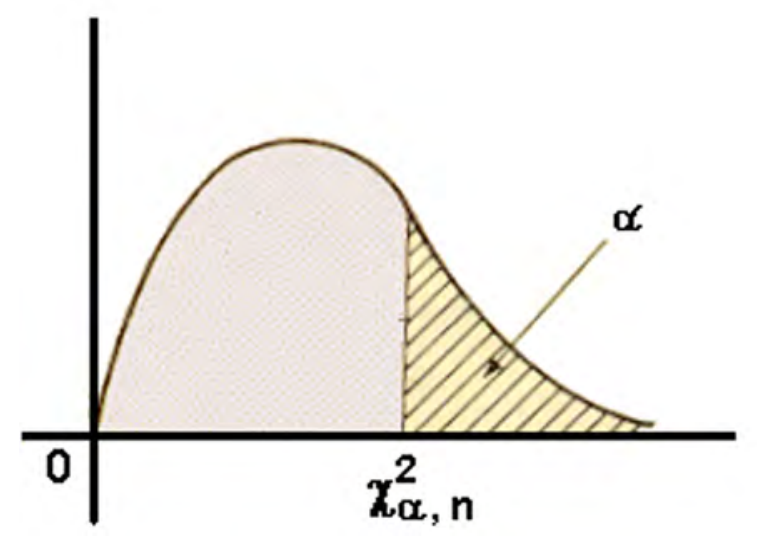

De acuerdo a la tabla para valores de chi cuadrado crítico de un valor superior de Alfa ( $a$ ) de 0,05 con un grado de libertad de 2 obtenemos 5,99.

Reemplazando valores:

$X$ g.l; $\alpha=X 2 ; 0,05=5,99$

El valor señalado se interpreta como la probabilidad de obtener un valor mayor que 5,99 es 0,05

Por lo tanto de acuerdo a la formula Chi cuadrado:

$$
\begin{aligned}
\text { Xexp. } & \geq X 2 ; 0,05 \\
84,62 & \geq 5,99
\end{aligned}
$$

Las variables no son independientes entre sí.

> Por lo tanto, se acepta la hipótesis

$\mathrm{HI}=$ Hipótesis General Alterna:

"Nos demuestra que el uso adecuado de los presupuestos a nivel de la Cadena de Hoteles San Luis, incide en la mejora de la Rentabilidad".

De acuerdo a los resultados se comprueba que las hipótesis son dependientes entre sí, por lo cual se comprueba que los resultados de rentabilidad asumidos por la empresa durante los años 2012 al 2016 han sido óptimos conforme al uso del presupuesto como instrumento financiero utilizado.

A mayor precisión, los resultados expuestos en el presente trabajo respecto de los Hoteles A, B y C de la cadena de Hoteles San Luis, demuestran que el presupuesto es una herramienta financiera muy importante, pues su ejecución disciplinada, posibilitó alcanzar la rentabilidad deseada, en mérito al cual la organización se viene afianzando, otorgando mayores ventajas a sus operadores, lo que le permitirá ser más competitiva.

\section{DISCUSIÓN}

La estrategia de administración financiera de la Cadena de Hoteles San Luis durante el periodo de análisis a través del uso de los presupuestos demuestra la consistencia de su aplicación al posibilitar que los objetivos de rentabilidad se vean alcanzados. Esta situación, nos 
permite demostrar la importancia del presupuesto, como una herramienta financiera necesaria que coadyuva a una adecuada gestión, mejorando no solo la rentabilidad de las empresas hoteleras, sino contribuyendo a garantizar el buen servicio ofertado inicialmente como un intangible.

Los presupuestos, al formar parte de la actividad empresarial de servicios hoteleros permiten una eficiente gestión presupuestal de ingresos por hospedaje, servicios de alimentación y turismo, posibilitando con ello el incremento de rentabilidad por posicionamiento del mercado hotelero; por fidelización de clientes; y por calidad del servicio, contribuyendo de esta manera a mejorar todas las operaciones de la organización, ayudándolas en el logro de sus objetivos, coadyuvando con el accionar de todos los entes ejecutivos; y por ende a desarrollarse en un marco de disciplina y formalidad, avanzar sin mayores riesgos, mejorando el proceso en un marco de eficiencia y eficacia.

El uso de este instrumento de ejecución financiera demuestra la relación de los presupuestos en la programación de inversión a través del mejoramiento de servicios y adecuada presentación de la infraestructura de la empresa, así como también permite una eficaz programación de recursos humanos, programación de compras e inversiones posibilitando un incremento de rentabilidad por reducción de gastos corrientes, reducción de costos y mejora de la razón costo beneficio.

Existe una relación direccional entre los procedimientos de auditoría de gestión y el tratamiento oportuno en la presentación de desviaciones presupuestales que podrían suscitarse durante la gestión. Esta relación, es la que ha permitido a lo largo del período materia de análisis adoptar los correctivos inmediatos posibilitando que no se afecte los resultados esperados, de tal forma garantizar los objetivos planteados y por ende el incremento del patrimonio de la organización.

Asimismo, esta disciplina en observancia de ambas herramientas de gestión, mejoraron los niveles no solo de organización de la empresa, sino lo que es más importante y que permite el crecimiento sostenido a lo largo de estos años, la satisfacción plena de los clientes.

\section{Agradecimiento}

Agradezco a la Dra. Jeri Gloria Ramón Ruffnner de Vega y a mi tutor Dr. Alberto B. Espinoza Valenzuela, ambos por su motivación y enseñanza permanente, que posibilitaron el inicio y culminación del presente trabajo de investigación, que me otorga la oportunidad de seguir avanzando en mis conocimientos académico profesionales.

\section{REFERENCIAS BIBLIOGRÁFICAS}

Acerenza, M. (1991). Administración del Turismo - Conceptualización y Organización. México D.F.: Editorial Trillas.

Bermúdez, M. (1995). El Hotelero: Cómo Ser El Mejor Anfitrión. Bogotá- Editorial Interamericana S.A. pp. 195 196.

Bernal, C. (2006) Metodología De La Investigación. México D.F: Pearson. Educación.

Bunge, M. (1997). La Investigación Científica. Barcelona: Editorial Ariel.

Burbano, Ortiz (1997). Enfoque Moderno De Planeación Y Control De Recursos. Santafé De Bogotá. Bogotá.

Cárdenas, R. (2008). Presupuesto, Teoría y Práctica. México D.F.: Editorial Mc Graw Hill.

De La Torre, F. (1990). "Introducción Al Estudio Del Turismo”, Editorial Trillas, México,

Fernández, P. (1970). Economía Y Gestión De La Empresa, Madrid: Ediciones Ice.

Gallegos, J. (2007). Gestión De Hoteles. Madrid: Editorial Paraninfo S.A. pp. 79-88.

Hans, R. (1961). Experience and Prediction. University of California Press. United State. 196. Traducción De Luis Piscoya Hermoza.

Hauyón, J. ((1968) "Perú: Proyecto Nacional De Turismo", Universidad San Martín De Porras, Lima - Perú,

Robbins y Coulter, (2005). Administración. México.

Hirschi T. (1969,) Obra La Teoría Del Control. Miami. Ediciones Ice.

Navas Y Guerras, (1996), La Dirección Estratégica De Las Empresas, Teoría Y Aplicaciones. Madrid: Civitas. 
Namakforoosh, M. (2005). Metodología De La Investigación. México: Editorial Limusa. 2da. Ed.

Decreto Supremo $\mathrm{N}^{\circ}$ 001-2015-MINCETUR, (09. JUN.2015), Reglamento de establecimientos de hospedaje.

Vasques, C., Cypriano, P., \& L'Hopital, F.. (2016). Previa del Panorama de la Hotelería Sudamericana. septiembre 6, 2016, de HVS Sitio web: https://www.hvs.com/arti- cle/7787-previa-del-panorama-de-la-hoteleria-sudamericana

Suarez A. (1970). Decisiones óptimas de Inversión y Financiación en la Empresa. España.

Ríos Gonzáles Cristóbal, Manual de Costos, Presupuestos y Adquisiciones y Abastecimiento. México, DF. Cengage Learning Editores, SA. 2007. 